\title{
The message for World Kidney Day 2009: hypertension and kidney disease-a marriage that should be prevented
}

\author{
George L. Bakris · Eberhard Ritz • \\ on behalf of the World Kidney Day Steering Committee
}

Received: 13 December 2008

(C) Japanese Society of Nephrology 2009

\section{Introduction}

The kidney is both a cause and victim of hypertension. High blood pressure is a key pathogenetic factor that contributes to deterioration of kidney function. Presence of kidney disease is a common and underappreciated preexisting medical cause of resistant hypertension [1]. Therefore, treatment of hypertension has become the most important intervention in the management of all forms of chronic kidney disease (CKD). For this reason, the forthcoming World Kidney Day (WKD) on 12 March 2009 will emphasize the role of hypertension for renal disease.

\section{How does one recognize the presence of chronic kidney} disease?

In contrast to a decade ago, today most laboratories around the world report estimated glomerular filtration rate (eGFR) instead of or in addition to serum creatinine. This now provides the physician with information about kidney

World Kidney Day 2009 Steering Committee: William Couser, Paul Beerkens, Co-Chairmen. Tom Reiser, Jan Lantink, Project Directors. Georgi Abraham, Alan Collins, John Feehally, Joel Kopple, Philip Li, Miguel Riella, Bernardo Rodriguez-Iturbe, Anne Wilson.

\section{G. L. Bakris $(\square)$}

Department of Medicine, Hypertensive Diseases Unit,

Pritzker School of Medicine, University of Chicago,

Chicago, IL, USA

e-mail: angela@worldkidneyday.org

\section{E. Ritz}

Department of Medicine,

Ruperto Carola University of Heidelberg,

Heidelberg, Germany

function that is, in general, more informative. As a result, a greater percentage of patients with diabetes or hypertension and their physicians have a better knowledge of their kidney function. Assessment of eGFR as an index of kidney function should be complemented by assessing urine for protein or albumin (preferred).

In spite of these laboratory updates, recent data demonstrate that a given patient's knowledge that he or she has CKD is very low. In a recent analysis of almost half a million people in Taiwan who took part in a standard medical screening program, $12 \%$ had CKD [2]. It was noteworthy that less than $4 \%$ of those with CKD were aware of their condition. People with CKD are several times more likely to die from cardiovascular (CV) causes than those without CKD; thus, hypertension is a major risk factor in this context [3]. The combination of CKD and hypertension, therefore, is a major public health issue; because of the costly treatments necessary for end-stage renal disease (ESRD), end-stage CKD has also become a substantial burden to health budgets.

\section{What is the worldwide frequency of chronic kidney disease?}

The frequency of CKD continues to increase worldwide, as does the prevalence of end-stage renal disease (ESRD) [4, 5]. The most common, but not only, causes of CKD are hypertension and diabetes. The presence of CKD is associated with a large increase in cardiovascular (CV) risk. Moreover, CV risk increases proportionally as eGFR falls below $60 \mathrm{ml} / \mathrm{min}$. Lastly, death from $\mathrm{CV}$ causes is higher in CKD and much higher than is cancer in CKD; as a result, the identification and reduction of CKD have become public health priorities [6]. 
The reported prevalence of CKD stages 1-4 in the most recent NHANES (national health and nutrition examination survey) between 1999 and 2006 was 26 million out of a population base of approximately 200 million. This represented United States residents aged 20 and older adult; of these, $65.3 \%$ had CKD stage 3 or 4 . Those with diabetes and hypertension had far greater prevalence of CKD (37 and $26 \%$, respectively) compared to those without these conditions (11 and 8\%, respectively) [7].

The most recent report of the United States Renal Data System estimates that nearly one-half million patients in the US were treated for ESRD in the year 2004 [8], and by 2010 this figure is expected to increase by approximately $40 \%$. Patients destined to progress to ESRD, i.e., the elderly, are a growing segment of the population. Additionally, males and African-Americans with pre-existing hypertension and CKD are also at much higher risk for ESRD [9]. This observation has also been confirmed throughout the developed world: Europe, Asia, Australia, and regions of India and Africa [4, 5].

\section{The role of hypertension}

Hypertension is a global problem, and the situation is projected to get worse. It is the major risk factor for development and progression in non-diabetic and diabetic CKD.

The world population is getting older, and aging is the most common risk factor for the development of hypertension and diabetes as well as CKD. Nearly 1 billion people worldwide have high blood pressure (defined as $>140 / 90 \mathrm{mmHg}$ ), and that number is expected to increase to 1.56 billion people by 2025 [10]. The prevalence of hypertension is predicted to increase by $24 \%$ in developed countries and by $80 \%$ in developing regions, such as Africa and Latin America. One report noted that 333 million adults in economically developed regions, such as North America and Europe, had high blood pressure in 2000, and an additional 639 million people in developing countries have this condition.

In 1999-2006, the prevalence of hypertension in US adults was $43.4 \%$ when defined as $>140 / 90 \mathrm{mmHg}$, and similar figures have been reported from many Western countries [9]. The rates of hypertension were highest in participants who were 60 years or older, i.e., $68-80 \%$ versus $25 \%$ in those between 20 and 39 years, in nonHispanic blacks (53\%) versus Caucasians (43\% versus Mexican-Americans (34\%). Furthermore, hypertension was more common in individuals with a higher body mass index $(\mathrm{BMI})(60 \%$ for BMI $\geq 35$ vs. $32 \%$ for BMI of 23$)$. Slightly more than half of adults with hypertension were aware of their disease in 1999-2004; fewer than half were treated for their hypertension with medications; less than two-thirds were controlled to $<140 / 90 \mathrm{mmHg}$ with medication [9]. This trend in poor blood pressure control is observed worldwide.

The hypertension control rate is substantially less in patients with CKD, particularly those with diabetes and CKD [1, 9]. This is illustrated by the National Kidney Foundation's (USA) Kidney Early Evaluation Program (KEEP), a US-based health-screening program for individuals at high risk for kidney disease [9]. The prevalence (86.2\%), awareness $(80.2 \%)$ and treatment $(70.0 \%)$ of hypertension in the screened cohort were high; however, blood pressure control rates were low (13.2\%). The proportion of hypertensive patients increased with advancing stages of CKD.

\section{Which blood pressure component is most relevant for renal and cardiovascular risk, systolic or diastolic?}

There is now consensus, based on the totality of the data, that systolic rather than diastolic blood pressure poses the greater risk for cardiovascular events and kidney disease progression. Against this background, it is relevant that in the KEEP study elevated systolic blood pressure accounted for the majority of patients with inadequate control. Male gender, non-Hispanic black race, and BMI of $30 \mathrm{~kg} / \mathrm{m}^{2}$ or more were inversely related to blood pressure control.

What is the blood pressure target for CKD patients? According to the different guidelines published by the major kidney societies, systolic blood pressure should be lowered to values $<130$ or $125 \mathrm{mmHg}$ if greater than $1 \mathrm{~g} /$ day of proteinuria is present. One has to be aware, however, that as a predictor of adverse CKD or cardiovascular events, office blood pressure may be inferior compared to ambulatory blood pressure measurement [11]. This issue is particularly relevant in CKD because of the tendency for nighttime blood pressure to be elevated (little or no nocturnal dip in blood pressure) and the fact that central (aortic) blood pressure tends to be higher than peripheral (brachial) blood pressure [11, 12]. In patients with diabetes, guidelines all recommend that lower blood pressure targets may provide further benefit, but prospective trials have thus far failed to confirm this epidemiological observation.

\section{The role of diabetic nephropathy}

As indicated above, diabetes and hypertension are the most common causes of CKD. There are currently over 240 million people with diabetes worldwide. This figure is projected to rise to 380 million by 2025 , largely due to 
population growth, aging, urbanization, unhealthy eating habits, increased body fat, and a sedentary lifestyle. By 2025 , the number of people with diabetes is expected to more than double in Southeast Asia, the Eastern Mediterranean and Middle East, and Africa. It is projected to rise by nearly $20 \%$ in Europe, $50 \%$ in North America, $85 \%$ in South and Central America, and $75 \%$ in the Western Pacific region. The top five countries with the highest prevalence of diabetes in order include India, China, the US, Russia, and Japan. Worldwide, more than $50 \%$ of people with diabetes are unaware of their condition and are not treated.

The same behaviors that increase obesity are shared with those predisposed to diabetes, i.e., family history, presence of hypertension, aging, excess body weight, lack of exercise, and unhealthy dietary habits. It is important to identify these risks early to reduce the development of diabetes and CKD, since CKD greatly amplifies the risk of cardiovascular events in the diabetic patient.

\section{The remaining challenge}

Under-diagnosis and under-treatment of CKD are worldwide problems: not only is CKD awareness low worldwide, but the relative lack of CKD risk factor awareness by physicians, i.e., hypertension and diabetes, is even more disturbing. Moreover, even awareness of these risk factors does not ensure adequate treatment; this could relate either to the behavior of the patient, the provider, or both. Thus, the problem of CKD remains a challenge as exemplified by recent data showing that between 1999 and 2006, <5\% of people with an eGFR $<60 \mathrm{ml} / \mathrm{min} / 1.73 \mathrm{~m}^{2}$ and proteinuria were aware of having CKD; of those with CKD stage 3 , awareness was only 7.5\%; for stage 4, awareness was less than $50 \%$. Awareness rates among those with CKD stages 3 or 4 were higher if co-morbid diagnoses of diabetes and hypertension were present, but even then, they were quite low (20 and $12 \%$, respectively).

One barrier to overcome in order to ensure greater awareness is a more focused education of physicians, since they are the purveyors of the patients' medical condition. In one survey, more than one-third of primary care physicians in the US were not aware that family history was a risk factor for CKD, while almost onequarter did not perceive African-American ethnicity as a CKD risk factor; in contrast, nearly all perceived diabetes $(95 \%)$ and hypertension $(97 \%)$ as risk factors for CKD. Even more problematic was the fact that while diabetes and hypertension were acknowledged as CKD risk factors, the achieved control rates (defined as reaching guideline goals) sadly remains well below 50\% among those treated.
What can be done about this problem?

There have been many consensus panels over the past decade to approach ways to achieve better blood pressure control and educate physicians to the stages of CKD [13, 14]. The road to improving outcomes is to focus on public awareness and screening programs as well as programs to educate both patients and physicians. Data from the KEEP screening program in the US have also indicated that blood pressure values are most likely to be at goal once a patient is aware they have kidney disease [15]. Data from Bolivia highlight the observation that once kidney disease is diagnosed, more appropriate interventions to reduce CKD risk factors such as hypertension are instituted [13].

Programs to address these issues have started around the world, including KEEP-type programs. As a major focus of World Kidney Day this year, the issue is hypertension in CKD (http://www.worldkidneyday.org).

Because of the aging world population and consequent increasing prevalence of hypertension and diabetes, CKD rates will continue to increase. This has and will continue to place an undue economic burden on societies given the costs for an ESRD program. In 2005, the US spent \$32 billion dollars on such programs. These facts mandate that measures be put forth to ensure timely detection and prevention of CKD progression. The key to ensure successful prevention of CKD is screening for hypertension, improved testing and diagnosis of predisposing co-morbidities such as diabetes and aggressive treatment to guideline goals.

The International Society of Nephrology (ISN) and the International Federation of Kidney Foundations (IFKF) have an ambitious long-term goal that worldwide every individual, particularly the patient with diabetes, knows his or her blood pressure values. Additionally, they should be aware that prompt treatment is necessary once blood pressure values are no longer in the normal range. Finally, our societies strongly encourage public health authorities to support efforts to raise public awareness of CKD and promote moves to reduce the risk of developing hypertension. Such governmental public health initiatives are exemplified by countries like the UK, Finland, and Japan reducing salt in the diet and mandating labels have sodium content as in the US. These initiatives have proven highly successful based on reduction in cardiovascular mortality and morbidity.

\section{References}

1. Sarafidis PA, Bakris GL. State of hypertension management in the United States: confluence of risk factors and the prevalence of resistant hypertension. J Clin Hypertens (Greenwich). 2008;10: 130-9. 
2. Wen CP, Cheng TY, Tsai MK, et al. All-cause mortality attributable to chronic kidney disease: a prospective cohort study based on 462293 adults in Taiwan. Lancet. 2008;371:2173-82.

3. McCullough PA, Jurkovitz CT, Pergola PE, et al. Independent components of chronic kidney disease as a cardiovascular risk state: results from the Kidney Early Evaluation Program (KEEP). Arch Intern Med. 2007;167:1122-9.

4. Atkins RC. The epidemiology of chronic kidney disease. Kidney Int Suppl. 2005;94:S14-8.

5. Alebiosu CO, Ayodele OE. The global burden of chronic kidney disease and the way forward. Ethn Dis. 2005;15:418-23.

6. Rosamond W, Flegal K, Furie K, et al. Heart disease and stroke statistics-2008 update: a report from the American Heart Association Statistics Committee and Stroke Statistics Subcommittee. Circulation. 2008;117:e25-146.

7. Ostchega Y, Yoon SS, Hughes J, Louis T (2008) Hypertension awareness, treatment, and control-continued disparities in adults: United States, 2005-2006. NCHS Data Brief. http://www. cdc.gov/nchs/data/databriefs/db03.pdf 1-8.

8. Coresh J, Selvin E, Stevens LA, et al. Prevalence of chronic kidney disease in the United States. JAMA. 2007;298:2038-47.
9. Sarafidis PA, Li S, Chen SC, et al. Hypertension awareness, treatment, and control in chronic kidney disease. Am J Med. 2008;121:332-40.

10. Kearney PM, Whelton M, Reynolds K, et al. Global burden of hypertension: analysis of worldwide data. Lancet. 2005;365:217-23.

11. Peterson GE, de BT, Gabriel A, et al. Prevalence and correlates of left ventricular hypertrophy in the African American Study of Kidney Disease Cohort Study. Hypertension. 2007;50:1033-9.

12. Townsend RR. Analyzing the radial pulse waveform: narrowing the gap between blood pressure and outcomes. Curr Opin Nephrol Hypertens. 2007;16:261-6.

13. Perico N, Plata R, Anabaya A, et al. Strategies for national health care systems in emerging countries: the case of screening and prevention of renal disease progression in Bolivia. Kidney Int Suppl. 2005;97:S87-94.

14. Whelton PK, Beevers DG, Sonkodi S. Strategies for improvement of awareness, treatment and control of hypertension: results of a panel discussion. J Hum Hypertens. 2004;18:563-5.

15. Rao MV, Qiu Y, Wang C, Bakris G. Hypertension and CKD: Kidney Early Evaluation Program (KEEP) and National Health and Nutrition Examination Survey (NHANES), 1999-2004. Am J Kidney Dis. 2008;51:S30-7. 